\title{
How Professionals Read Online Documents to Formulate Recommendations
}

\author{
M:S. Melenhorst \\ University of Twente, the Netherlands \\ m.s.melenhorst@utwente.nl
}

\author{
T.M. van der Geest \\ University of Twente, the Netherlands \\ t.m.vandergeest@utwente.nl
}

\begin{abstract}
Professionals often read with the purpose of formulating a judgment based on what they have read. Increasingly, the documents to be used are available electronically. This adds to the complexity of the task.

In the presented study seven participants read two sets of articles that were used to formulate recommendations regarding two technical communication issues.

Readers proved to be not only driven by their judgment task, but by the articles they were reading as well. This strategy reduced reading efficiency. An electronic notepad was used to store copied citations from the articles. Readers evaluated these citations superficially during reading, while they evaluated them more thoroughly when the recommendations had to be written.
\end{abstract}

\section{Introduction}

Professionals are often confronted with large document collections that they have to read as the basis for a judgment like a recommendation or a decision. The documents in these collections usually contain contradictory information, they are lengthy and the authors have purposes that do not coincide with those of the readers. Reading and evaluating these documents, often referred to as reading-to-assess; is a challenging task that demands a high share of the available cognitive resources.

Previous research showed that reading-to-assess is highly active and purpose-driven. Professionals read very selectively. For instance, representatives sometimes only read $3 \%$ of a policy document. Nevertheless they are able to ask critical questions during a parliamentary debate [1]. Information is evaluated critically and integrated with prior knowledge [2]. In tum, prior knowledge directs the reading process: readers formulate hypotheses for which confirmation is sought in the document collection [3].
Thanks to the digital revolution, documents are increasingly available online or on CD-ROM. These digital documents are often stored in hypertext format, which gives the reader the ample opportunity to select the information they need for their judgment task. At the same time, this makes the reading-to-assess task more complicated, because navigating through information space can cause cognitive problems like a lack of overview of the information available and its location in the set of documents [4].

How do readers cope with the challenges imposed by the online environment? How do they manage to collect information from documents that contradict each other while the time to read them is limited? How do they come to a substantiated decision? The study reported in this paper will cover these issues. The results have important implications for document designers.

\section{Method}

In order to answer our research questions we set up an experiment. Seven participants (Master students in Technical Communication) were given two tasks. In the first task we asked them to formulate recommendations on whether animations are appropriate to explain the effects of eating too much fat food to children. In this task the participants were given explicit criteria for their recommendations in the form of four questions that had to be addressed. In the second task, they had to formulate an advice on how to structure the homepage of a corporate intranet. The second task description did not contain explicit criteria. They could use a set of 5 and 4 articles respectively as the basis for their advice. The set contained articles of different genres that contradicted each other on some points. The set was presented in an online environment as if it were the result of query.

The articles were made accessible through a permanently visible navigation bar and through an article index that contained the full references to the materials. We added an interactive table of contents to each article. The participants could use an electronic Notepad without any restrictions. 
After explanation of the procedure and after reading the task description, participants could read and evaluate the documents for 30 minutes. They read and thought aloud, verbalizing everything that came to their mind. After 30 minutes or after deciding that they had read enough, the participants wrote their recommendations without time-restrictions.

\section{Results}

The results showed considerable differences between the reading processes. The most important differences can be traced back to the distinction between purpose-driven and text-driven reading. This topic will be addressed in the next section. Interactions with the online environment played an important role during reading. This aspect of reading will be addressed in 3.2 .

\subsection{Purpose-driven versus text-driven reading}

The participants adopted different strategies during reading. One particular participant was focused heavily on the task: she was deliberately looking for information on the specific issues asked for in the task description: "This doesn't address whether Flash is better for certain kinds of learning, which is what we're looking at". She was looking for evidence that confirmed her hypothesis that animations were good for showing processes. This hypothesis was based on her prior knowledge.

The majority of the other participants had a more general task conception, consisting of a few characteristic words from the task description, like 'animation' and 'children'. They read the information with these terms in mind, considering information relevant if it contained a term and skipping down if it did not ("Not worried, that's just about audio"). This strategy of condensing the task description to a few words helped the readers cope with their complex reading-to-assess task.

The participants' task conceptions were strongly related to their reading strategies: the more specific the task conception, the more purpose-driven the reading strategy. Readers that had a specific task conception, selected information based on the task more often than readers with a condensed task conception.

When the task conception was less specific, the articles influenced the reader to a larger extent than the task. Participants changed their perspective on the task, depending on specific relevant information in the articles.

The articles were not written to use as the basis for recommendations. The necessary transformation from the article to the advice task did not take place, when participants had a condensed task conception. With phrases like "That's good" or "Interesting" they evaluated the relevance of the information, but they did not comment on whether the information could be used as arguments for their recommendations.

\subsection{Interacting with the online environment}

In contrast to previous research the participants in this experiment read within an online environment. Selecting relevant information from the article set and from individual articles required much effort from the readers.

To select articles, they used the permanently visible navigation bar. Most participants did not explain why they selected a particular article: they read the articles one by one. Only one participant explicitly motivated her selections: for example, when selecting an article on instructional use of multimedia, she stated: "Let's see if this deals with the multimedia issue". Thus, her reading purposes guided her selection decisions.

Within an article, readers used an interactive table of contents that was positioned at the top of each article as well as the scroll bars to select relevant information. Participants scanned the table of contents looking for terms from the task description. When they encountered one of these terms, they jumped to the corresponding section by clicking on its title. After evaluating its content on relevance, participants browsed through the article by using the scroll bars, looking for additional relevant information. Thus, information is selected based on the occurrence of keywords.

The articles' structure was an important determinant for the amount of effort the readers put into retrieving the most relevant information. Articles with a structure familiar to the readers offered opportunities to retrieve relevant information quickly. For example, in the second task we provided the readers with a conventionally structured research article. Participants immediately jumped to the conclusions and on some occasions to the method, retrieving relevant information quickly.

On the other hand, articles with a complicated or unknown structure discouraged readers to evaluate them thoroughly. For instance, the article set in the second task contained an article that was a combination of a column and a set of guidelines. One participant browsed through the article and stated: "This is a little hard to read (...) There are no conclusions". The lack of conclusions made her decide to proceed with the next article.

As five lengthy articles contained too much information to remember until the recommendations had to be written, the electronic Notepad was used frequently. The majority of the participants used it to store copied citations from the articles which contained terms from the task description. This can be considered as a preliminary evaluation of relevance. 
By copying citations that were possibly relevant and useful for their recommendations, the readers postponed a more thorough evaluation until the recommendations had to be written. Only when formulating their recommendations most participants evaluated whether these citations could be used as arguments.

Some participants, however, processed the information deeper already during reading. They wrote down summarizing keywords, paraphrases, or draft recommendations. They structured these self-formulated notes according to their own categories or according to the questions from the task description.

\section{The quality of recommendations}

We want to relate the quality of the recommendations to the readers' approach in order to determine which characteristics of the reading process support good advice. Therefore we will ask experienced web designers to sort the recommendations in terms of completeness (has every issue from the task description been addressed satisfactorily?), persuasiveness (are the recommendations convincing?) and applicability (are they immediately applicable in practice?). In addition, we will ask them what constitutes good advice in their opinion. If they agree on what are good recommendations, we might be able to relate advice quality to characteristics of the reading process.

This study is still ongoing at the time of writing. We will give an overview of the results in our presentation.

\section{Discussion and conclusions}

The results presented above have important implications for document design. These implications are discussed in this section.

In contrast to existing research (in particular Bazerman, 1985), we found that readers are not only purpose-driven, but are guided by the articles as well. It appears to be related to the reduction of the task description to a few terms. Besides, they evaluated information during reading only superficially. Using the notepad extensively, they postponed a more detailed evaluation of relevance and usefulness until the recommendations had to be written. This approach is less efficient than when readers evaluate information already during reading.

These characteristics of the reading process are considered strategies to reduce the complexity of the reading-to-assess task. How can document designers help readers focus on the task? How can they help them cope with different author and reader purposes and an online environment that makes a fast selection of relevant information a challenge?

As a first possibility, document designers should use well-known structures and signal them to the readers by means of, for instance, an informative table of contents.

Second, document designers may use the online environment. Multiple access structures tailored to the various reader purposes reduce the complexity of the reading-to-assess task. Customized document structures may make the selection and evaluation of information that is relevant and useful much easier.

A third suggestion might be to improve note-taking by providing a tool in which readers can structure information according to the criteria for the judgment. This might help readers focus on the task.

Future research is necessary to evaluate the effects of these tools on the reading process so that we can help to improve the efficiency and effectiveness of the reading process.

\section{References}

[1] Neutelings, R. \& Pander Maat, H. (1997). Investigating the Processes of Reading-to-Assess Among Dutch Legislators. Journal of Literacy Research, 29 (1), p. 47-71.

[2] Bazerman, C. (1985). Physicists Reading Physics. Schema-Laden Purposes and Purpose-Laden Schema. Written Communication, 2(I), p. 3-23

[3] Duyne, P.C. van (1983). Beslissen in eenvoud. [Simply deciding] Arnhem: Gouda Quint B.V.

[4] Geest, T.M. van der (1994). Hypertext: writing and reading in a non-linear medium. In Steehouder, M., Jansen, C., Poort, P. van der \& Verheijen, R. (eds.) Quality of technical documentation. Utrecht: Utrecht Studies in Language and Communication 